\title{
Transformaciones y retos epistemológicos, políticos y sociales en las culturas alternativas menstruales
}

\author{
Epistemological, political and social transformations \\ and challenges in menstrual alternative cultures
}

MIREN GUILLÓ ARAKISTAIN ${ }^{1}$ (Grupo de Investigación en Antropología Feminista AFIT, Universidad del País Vasco UPV/EHU) ${ }^{2}$

Artículo recibido: 14 de febrero de 2021 Solicitud de revisión: 26 de marzo de 2021 Artículo aceptado: 5 de noviembre de 2021

Guilló Arakistain, Miren (2022). Transformaciones y retos epistemológicos, políticos y sociales en las culturas alternativas menstruales. Recerca. Revista de Pensament $i$ Análisi, 27 (1), pp. 1-24. doi: http://dx.doi.org/10.6035/recerca.5762

Resumen

Este artículo, que se basa en una investigación etnográfica, se centra en el análisis de las características sociales, políticas y emocionales de las políticas y culturas alternativas de la menstruación, así como en los cambios que están sucediendo en torno a la percepción y la experiencia menstrual. Para ello, tiene como objetivo atender la intensificación y la diversificación de las propuestas en las últimas décadas y las diversas estrategias político-sensoriales seguidas por sus protagonistas, así como las ideologías feministas y de salud que subyacen y se generan en ellas. De esta manera, se pretende profundizar en el activismo menstrual, partiendo de la idea de que este análisis puede servir, además, para identificar y estudiar transformaciones sociales más generales. El artículo concluye proponiendo algunos retos epistemológicos, políticos y sociales para seguir indagando en dichas culturas alternativas y en su potencial para cuestionar las desigualdades sociales.

Palabras clave: cuerpo, feminismo, afectos, subjetividades políticas, menstruación, culturas alternativas menstruales.

\footnotetext{
${ }^{1}$ miren.guillo@ehu.eus

${ }^{2}$ Agradezco a Mari Luz Esteban, Laura Muelas e Irantzu Fernández sus aportaciones a este artículo y, especialmente, a todas las personas entrevistadas que han participado en la investigación.
} 
Abstract

This article, which is based on an ethnographic research, focuses on the analysis of the social, political and emotional characteristics of the alternative politics and cultures of menstruation, as well as the changes that are happening around the perception and experience of menstrual. For this, the article, aims to address the intensification and diversification of menstrual proposals in recent decades and the various political-sensory strategies followed by their protagonists, as well as the feminist and health ideologies that underlie and are generated in them. In this way, it is intended to delve into menstrual activism, from the idea that this analysis can also serve to identify and study more general social transformations. The article concludes by proposing some epistemological, political and social challenges to continue investigating these alternative cultures, and in its potential to question social inequalities.

Key Words: body, feminism, affects, political subjectivities, menstruation, alternative cultures of menstruation.

\section{INTRODUCCIÓN}

El surgimiento de nuevas culturas menstruales produce nuevas configuraciones corporales, dando lugar a experiencias encarnadas que convierten la menstruación en un campo de disputa y transformación social. Las ciencias biomédicas funcionan como dispositivos legitimados y legitimadores de producción de conocimiento científico sobre el cuerpo, y la explicación hegemónica del ciclo menstrual contribuye a la generización del mismo, afectando a las vivencias y las identidades sociales de las personas y contribuyendo a las desigualdades sociales y de género. Los procesos de patologización y estigmatización menstrual se constituyen a partir de diversas ideologías simbólicoculturales, del paradigma biomédico y de las actuaciones de las corporaciones relacionadas con la industria menstrual, constituyendo, así, lo que en otros trabajos he llamado la ideología de la normatividad menstrual (Guilló-Arakistain, 2020).

No obstante, dicha normatividad es discutida a través de diferentes políticas y culturas que desestabilizan y subvierten dicho paradigma biomédico, dando lugar, en las dos últimas décadas, a la proliferación de un sinfín de propuestas menstruales en diversos lugares, también en el contexto del Estado español. Se constituye así un cuerpo político feminista (Esteban, 2011) múltiple de la menstruación, que se materializa a través de prácticas e ideologías que cuestionan la medicalización y la patologización de la visión biomédica occi- 
dental del ciclo, mediante diferentes procesos e iniciativas críticas, como, por ejemplo, distintas maneras alternativas de gestionar el sangrado y dispositivos feministas para mejorar las vivencias en torno al ciclo, talleres y encuentros, campañas, fanzines, murales, performances, etc. Valores y prácticas que se generan en contraposición a la visión hegemónica menstrual, como ámbito de resistencia y agencia, donde la dimensión emocional juega un papel muy importante, facultando procesos de transformación social y política.

Así, este artículo, que se basa en una investigación etnográfica sobre políticas y culturas alternativas menstruales llevada a cabo entre los años 20082019, trata de profundizar en dichas propuestas y en los cambios sociales que implican, atendiendo a su aumento y diversificación, a las estrategias políticosensoriales empleadas y las ideologías sobre el género y la salud que subyacen y se generan en ellas. Para ello, primero se ahondará en los anclajes conceptuales, genealógicos y contextuales de dichas políticas corporales. Posteriormente, se expondrán los aspectos metodológicos de la investigación, para pasar después a profundizar en algunos hallazgos relacionados con 1) el aumento y la diversificación de dichas propuestas, 2) los procesos afectivos y de socialización y las estrategias políticas-sensoriales, así como 3) las diferentes ideologías feministas y en torno al género que las conforman. El artículo concluye proponiendo algunos retos epistemológicos, políticos y sociales de las culturas alternativas menstruales.

\section{EL CUERPO COMO CAMPO DE DISPUTA Y RESISTENCIA: IDEOLOGÍA DE LA NORMATIVIDAD MENSTRUAL Y CULTURAS ALTERNATIVAS}

La trayectoria de los estudios antropológicos, sociales y feministas en torno al ciclo menstrual es un campo amplio donde han emergido diferentes visiones, desde perspectivas clásicas de la antropología de la menstruación, centradas mayormente en lecturas simbólicas y culturales —en las que se analizaban aspectos como la diversidad semántica de los tabúes menstruales-, a investigaciones más influenciadas por los desarrollos epistemológicos de la teoría social y antropología simbólica, médica y feminista, así como de cambios sociales y feministas a partir de los setenta. Poco a poco, en las últimas décadas se han ido revisando las perspectivas etno-androcéntricas tradicionales y buscando aproximaciones más contextualizadas, así como reparando más 
en las relaciones sociales, las vivencias corporales y la agencia de las mujeres y los cuerpos menstruantes, visibilizando su variabilidad intercultural. Actualmente, se multiplican los temas y las áreas de trabajo, del activismo a la justicia menstrual y de un lenguaje más inclusivo respecto a la identidad y las vivencias del género, lo que se refuerza, sobre todo, a partir del siglo XX. De hecho, entre toda la actual variedad de temas, cada vez más estudios prestan atención a las culturas alternativas y activismos menstruales. ${ }^{3}$

En esta investigación, que se ubica en y se nutre de estas y otras genealogías, además del término de origen anglófono de activismo menstrual, ${ }^{4}$ que hasta hace algunos años no se había utilizado apenas en el contexto español, otro concepto clave empleado en referencia a la articulación de dichas contraposiciones ha sido el de políticas y culturas alternativas de la menstruación, un concepto que considero que, dado el contexto temporal y cultural de esta investigación, tiene cierta apertura semántica e inclusiva, teniendo en cuenta la diversidad de características y de intensidad de dichas iniciativas en nuestro contexto en las últimas décadas. Mediante dicho término me he referido a todo tipo de prácticas, desde la gestión alternativa del ciclo menstrual, que implica la denuncia contra la industria menstrual o la socialización de otro tipo de dispositivos y alternativas para el sangrado y otras tecnologías feministas, hasta la realización de talleres y proyectos, fanzines, expresiones audiovisuales y artísticas, como performances, acciones y murales en la calle, robos colectivos de compresas y otras tantas creaciones componen algunas de las propuestas políticas más relevantes en las políticas y las culturas alternativas de la menstruación. Una característica fundamental es que en el centro de dichas iniciativas está la agencia feminista, individual y colectiva. Eso sí, dichas políticas se producen siempre dentro de contextos económicos, culturales y políticos concretos que las afectan pero que, a su vez, son afectadas por todas estas coyunturas, incidiendo en la conformación de las subjetividades y potenciando la capacidad de acción de los sujetos.

\footnotetext{
${ }^{3}$ Véanse, entre otros trabajos, Kissling, 2006; Bobel, 2010, 2015; Guilló Arakistain, 2013, 2014, 2020a; Persdotter, 2013; Felitti, 2016, 2017; Fahs, 2016; Ramírez, 2016, 2019; Blázquez y Bolaños, 2017; Tarzibachi, 2017; Gómez y Marco, 2020; Bobel et al., 2020.

${ }^{4}$ Para una genealogía del activismo menstrual en el contexto español, véase, por ejemplo, la trayectoria que recoge Irene Aterido (2018) en el monográfico de MyS (Mujeres y Salud) de la Red Caps, red que en su quehacer ha dedicado diferentes artículos, seminarios y cursos en línea sobre el ciclo menstrual. Véase, también, Blázquez y Bolaños, 2017; Guilló Arakistain, 2020a; Gómez y Marco, 2020; etc.
} 
En el contexto estatal, no solo se ha dado un aumento y una intensificación de las propuestas, sino que estas han adquirido una mayor visibilización, a través de las redes sociales y los medios de comunicación, influyendo mucho en su difusión. En décadas anteriores, y especialmente con el cambio de siglo, las pocas propuestas existentes eran aún más subalternas y menos visibles, aunque, según lo estudiado, muy significativas. Estas surgían en diferentes ámbitos, como en espacios de carácter terapéutico y espiritual, a través del cuestionamiento del estigma y del cultivo de vivencias e imaginarios menstruales más placenteros y habitables (Guilló Arakistain, 2018, 2020b); pero también en espacios más contraculturales, como centros sociales y gaztetxes, acampadas políticas o jornadas feministas. Las actividades que se desarrollaban en estos espacios eran mayoritariamente talleres para cuestionar la industria menstrual y el tabú y producir compresas de tela, fanzines o conocer la copa, además de performances o acciones callejeras (como las del colectivo de Sangre Menstrual), ${ }^{6}$ todo ello desde un carácter de denuncia, reivindicación y subversión, y desde una filosofía de autogestión colectiva DIY (Do It Yourself). Se trataba a menudo de iniciativas colectivas y, a veces, de autoría desconocida o anónimas, algo relacionado en general con la cultura política de la época. En la segunda década del siglo XXI se fueron multiplicando los talleres menstruales en espacios más institucionales, como, en el contexto vasco, en las denominadas escuelas de empoderamiento y en las casas de las mujeres, espacios abiertos donde el perfil de las participantes era variado. Si bien la norteamericana Chris Bobel (2010), en su pionero e inspirador trabajo sobre activismo menstrual, distinguió entre feministas espirituales y feministas radicales por sus diferentes modos de hacer y enfoques feministas, en el contexto del Estado español los perfiles se difuminan más, algo que también apuntan Emma Gómez Nicolau y Elisabet Marco Arocas (2020), diversidad relacionada con las propias características culturales, políticas y sociales.

A su vez, no podríamos entender dichas propuestas sin mencionar los activismos feministas de la salud que comenzaron a desarrollarse en el Estado a partir de los años setenta y ochenta del siglo pasado, especialmente las reivindicaciones ligadas a la salud sexual y reproductiva. Dicha época se caracterizó por procesos de toma de conciencia de las mujeres, la denuncia del androcentrismo del sistema médico y la construcción de conocimientos en torno a la

\footnotetext{
${ }^{5}$ Los gaztetxes son espacios autogestionados (muchas veces, ocupados) por la gente joven en el contexto vasco.

${ }^{6}$ https://sangremenstrual.wordpress.com/about/ (consultado el 10 de febrero de 2020)
} 
salud partiendo de metodologías colectivas, que pretendían impulsar el empoderamiento corporal y la autogestión (Esteban, 2001; Blázquez, 2005; Nogueiras, 2013; Epelde, Aranguren y Retolaza, 2015). Las formas de resistencia y subversión frente a los procesos de medicalización y patologización de los cuerpos surgidos en aquella época (Nogueiras, 2013) han influido en los activismos de la salud de las décadas posteriores, de manera más o menos explícita. Aunque las reivindicaciones y las iniciativas relacionadas con el ciclo previas a principios de siglo no se hayan apenas analizado - un capítulo invisible en la historia del feminismo, según Bobel (2010)_, existen relatos sobre algunas campañas, acciones o reflexiones feministas que incluían la menstruación. Estas iniciativas se desarrollaron mayormente en el ámbito de la salud, en particular en el marco de la filosofía del self-help y en proyectos y publicaciones más amplios (véase, por ejemplo, Taboada, 1978). Aquí no nos podemos extender en ello, pero es vital tener en cuenta las genealogías activistas y teóricas de las políticas menstruales en nuestros contextos.

A su vez, es preciso apuntar que, a la par que estas prácticas y teorías cambian, también la ideología hegemónica se transforma. Por ejemplo, la pluralidad de la práctica biomédica, mucho más diversa que el propio paradigma, refleja cambios sociales también diversos, de modo que conviven en dicho ámbito reivindicaciones y enfoques feministas con fuertes desigualdades sociales que se sustentan tanto en perspectivas conservadoras del género como en el sistema neoliberal, que perpetúa esas mismas desigualdades, enmarañadas con otras fuentes de opresión. Pero, además, en el contexto cultural neoliberal del pensamiento positivo (Ahmed, 2004; Ehrenreich, 2011; Illouz, 2014), el mercado menstrual instrumentaliza los discursos y las transformaciones feministas, mostrando imaginarios menstruales más positivos en su publicidad, pero presentando el tener que habitar el ciclo de manera positiva como responsabilidad totalmente individual, sin explicitar el contexto de desigualdad donde se produce dicho estigma (Przybylo y Fahs, 2020) y neutralizando las reivindicaciones feministas de la menstruación, algo que contribuye a constituir la misma hegemonía. Es por ello que propongo partir de un concepto complejo de la ideología de la normatividad menstrual, que contemple cómo se imbrican entre sí normas muy diferentes, incluso a veces contrapuestas, junto con una perspectiva crítica de la sociedad del consumo y un enfoque antiesencialista de los cuerpos menstruantes. 


\section{DESCRIPCIÓN DEL ESTUDIO Y ASPECTOS METODOLÓGICOS}

Como ya he apuntado, este artículo se basa en una investigación que pone en el centro del análisis las políticas y las culturas alternativas del ciclo menstrual, investigando las continuidades, las tensiones y las rupturas que se producen en ellas, tanto respecto a las ideologías biomédicas y culturales hegemónicas como en relación con la propia teoría feminista, analizando para ello las formas de gestionar y vivir la corporalidad, el género y el feminismo, tomando las prácticas corporales y los procesos vitales de las personas participantes en el estudio como punto de partida en todo el proceso.

Para ello se ha llevado a cabo una extensa etnografía corporal entre 2008-2019 basada tanto en una observación participante extensa, como en el análisis de itinerarios corporales (Esteban, 2004), construidos a través de entrevistas en profundidad repetidas en el tiempo a 36 personas implicadas en estas propuestas críticas. La mayoría de estas personas son feministas de entre 20 y 40 años, de diversos contextos urbanos y rurales, y que en sus trayectorias están labrando alternativas vitales en cuanto al género o la salud. La construcción de los itinerarios permite visualizar de modo complejo los cambios vitales de las personas en sus contextos, poniendo el énfasis en su dimensión corporal (Esteban, 2004). Teniendo en cuenta los cambios que se han producido en el ámbito de estudio, el hecho de volver a entrevistar y quedar con la mayoría de participantes en la última fase del estudio ha permitido profundizar tanto en sus cambios personales como generales, enfatizando su dimensión temporal. Además, se ha participado en 34 iniciativas diferentes (talleres, seminarios, encuentros, instalaciones...). Todo ello ha ido acompañado de una mirada auto-etnográfica y reflexiva como sustento del análisis, ya que la autora estaba implicada en el tema de estudio desde algunos años atrás por su propia experiencia menstrual y por su activismo feminista y menstrual y su experiencia como dinamizadora de talleres en este campo.

\section{EL CUERPO POLÍTICO MENSTRUANTE Y SUS RECONFIGURACIONES}

A lo largo de estos años he observado cómo las propuestas críticas que han ido surgiendo cuestionan la visión negativa hegemónica, la industria 
menstrual, el sistema biomédico y los valores culturales que refuerzan y retroalimentan el estigma social, buscando nuevos imaginarios alternativos. Para muchas de las personas que han participado en la investigación, resignificar el ciclo menstrual es una manera de incidir en la transformación social: implica denunciar que en la sociedad occidental y en el paradigma biomédico imperante determinados cuerpos valen más que otros, que el conocimiento científico médico se construye sobre esa base y que el mercado neoliberal y, en especial, la industria farmacéutica mercantilizan dichas desigualdades sociales.

\subsection{Aumento, diversificación y cambios en las propuestas menstruales}

Asimismo, al igual que sucede en el feminismo en general, también el perfil de las personas que se implican en estas propuestas se ha diversificado. De esta forma, en esta investigación se han identificado una serie de factores que pueden contribuir a entender dicho auge, tales como: la difusión de la cultura y los discursos feministas; el aumento de propuestas en torno al cuerpo, también en el ámbito feminista; perspectivas feministas surgidas alrededor de la violencia obstétrica; el auge de las medicinas alternativas, las terapias holísticas y la espiritualidad; los planteamientos en torno a la economía social y el consumo político; así como los propios efectos de la sociedad de consumo capitalista (Guilló Arakistain, 2020a: 258-259). Estos procesos sociales, aunque son diferentes entre sí, han incidido directamente en el desarrollo del activismo menstrual.

El tipo de propuestas que más han destacado han sido, sobre todo, los talleres y encuentros, así como todo lo relacionado con las tecnologías feministas del ciclo, en relación a espacios muy diversos, donde lo virtual tiene un protagonismo importante. Para muchas implicadas, además de diversas lecturas, estos dos campos han supuesto hitos importantes en sus vivencias corporales y afectivas, así como en la politización del tema.

En cuanto a las tecnologías feministas relacionadas con el ciclo menstrual, en los últimos tiempos se han socializado dispositivos más ecológicos, económicos y cómodos para la gestión del sangrado, así como diversas tecnologías para vivir mejor el ciclo, reducir los dolores o aumentar el autoconocimiento. Todo esto ha implicado profundos cambios en las vivencias corporales y la 
percepción menstrual de muchas. $\operatorname{Luna}^{7}$ (36), una informante implicada en diversas iniciativas sociales y en propuestas en torno al ciclo, el feminismo y la salud desde una perspectiva holística, subrayaba que en el instituto leía diferentes artículos sobre la salud, encarnando desde joven un enfoque crítico ante las injusticias y los discursos hegemónicos sobre el cuerpo. Ya era consciente entonces de los residuos que generaban las compresas y los tampones, así como de lo nocivos que son para la salud. Con veinte años, una chica que había conocido en campamentos le habló de las esponjas menstruales y poco después otra amiga le regaló una copa menstrual:

Y eso ya era la bomba; el invento del siglo [...] en aquella época hicieron un pedido por Internet, y así un porrón de ellas, empecé a utilizarla y una maravilla. Yo utilizo la copa y así aprovecho para olerlo [la sangre], para observarlo, qué textura tiene, lo he probado alguna vez por curiosidad. No lo uso tanto para diagnóstico, pero sí para ver qué textura tiene, qué color, qué olor... me da mucha información (Luna, 2018).

El uso de las compresas textiles, las esponjas, el sangrado libre y, sobre todo, la copa ha cambiado la experiencia vital de muchas. Para algunas, el uso de compresas de tela, sean fabricadas por ellas o compradas, ha sido otra manera de vivir la experiencia menstrual. Maialen (25), que trabaja en diversos proyectos feministas y del ámbito educativo y que participó en un grupo de trabajo sobre la menstruación, por ejemplo, para probar diversos dispositivos, decidió hacer compresas artesanales: «Son gratuitas, te las coses tú misma y es una manera de autogestionarte». Cuando entrevisté por primera vez a Nahia (32), una feminista que hace años había hecho una pieza artística sobre la regla, me dijo que no emplear ningún dispositivo le relajaba el cuerpo y los músculos, y muchas otras informantes, sobre todo los últimos años, han estado probando lo que se está denominando como sangrado libre.

Mediante argumentos ecológicos, económicos, prácticos y políticos, las participantes en el estudio han transmitido la necesidad de otro tipo de dispositivos, así como la búsqueda de otras alternativas en la vida: una dimensión de lo alternativo que, para ellas, se relaciona con lo comunitario, lo material, lo identitario y con prácticas del día a día. Así pues, a través de todas esas críticas y la decisión de realizar otro tipo de prácticas corporales, se encarna una socialización para el cambio. También existen en el mercado cada vez más compre-

\footnotetext{
${ }^{7}$ Los nombres empleados para las personas participantes de la investigación son ficticios para preservar su anonimato.
} 
sas y tampones elaborados con fibras sin blanquear o con fibras orgánicas, opciones más saludables y más respetuosos con el medioambiente. No obstante, cada argumento de los citados toma más o menos fuerza según el contexto o el carácter de cada proyecto.

De esta forma, la observación de las tecnologías es fundamental porque el diseño de artefactos y, en general, las políticas de la tecnología son un espacio productivo para la negociación del poder de las mujeres y los grupos subalternos (Layne, 2010; Hester, 2018). Linda Layne (2010) define las tecnologías feministas como herramientas y conocimientos que contribuyen a mejorar la capacidad de las mujeres para desarrollar, aumentar y expresar sus habilidades. Sin embargo, propone observar todas las consecuencias que implica cada tecnología, pues a veces puede que una tecnología empodere a determinadas mujeres, pero tal vez a costa de producir nuevas desigualdades sociales, por ejemplo, si dicha producción implica condiciones laborales injustas para otras o perspectivas reduccionistas del género.

Respecto a las tecnologías para el sangrado, es interesante detenernos a observar los procesos en relación con dispositivos como las copas, las esponjas o las compresas de tela, ya que tradicionalmente se ha impulsado la autoproducción y autogestión dentro de la filosofía DIY, así como un consumo más consciente y respetuoso con el medioambiente. Sin embargo, en los últimos años encontramos que, bajo la lógica del mercado neoliberal, también está presente este tipo de dispositivos, por ejemplo, cuando grandes corporaciones se han sumado a la producción de la copa, pero sin prestar atención al proceso ético y feminista que muchas pequeñas productoras habían puesto sobre la mesa (respecto a condiciones, materiales y procesos de producción, distribución, etc.). Otro caso controvertido sería el de las aplicaciones menstruales, que por un lado son una herramienta útil para el conocimiento del propio ciclo para muchas menstruantes (al igual que los diagramas, los calendarios menstruales, etc.), pero, por otro, capitalizan toda la información acumulada en dichas aplicaciones sin claros procesos de aprobación, a través de acuerdos entre distintas aplicaciones y diferentes empresas y universidades (Thiébaut, 2018). Por tanto, queda patente otro aspecto de una sociedad de consumo que rentabiliza la agencia de los cuerpos menstruantes, por lo que no podemos dejar de complejizar la pregunta sobre qué hace feminista a una tecnología y considerar todo tipo de consecuencias materiales posibles. 
Otras iniciativas habituales, como señalaba anteriormente, son los talleres y encuentros donde se suelen tratar temas que tienen que ver con las vivencias relacionadas con el ciclo, se discuten sus significados sociales y culturales androcéntricos o se comparten conocimientos sobre dispositivos para su gestión y la autogestión de la salud. Estos procesos colectivos que se dan en geografías muy diferentes (ciudades, pueblos, entornos rurales...) son espacios de seguridad y empoderamiento donde tienen lugar muy distintas emociones y se articulan perspectivas críticas y solidaridades entre las participantes. Es el tipo de iniciativa que más se ha repetido en la etnografía, precisamente por su carácter colectivo y por el clima de confianza y sosiego, así como por su accesibilidad (en cuanto al tiempo, a la economía o por la cercanía geográfica). El punto de partida para organizar cada taller puede ser muy diverso. Ana (zo), que forma parte de un colectivo feminista que ha organizado talleres, explica cómo sucedió en su caso: «Nace de una necesidad que teníamos en realidad nosotras. Nos pasamos mogollón de tiempo hablando de nuestras reglas [...] a veces no le damos importancia a todas las cosas que sabemos sobre nosotras».

También han destacado en dichos encuentros dimensiones más específicas, como la educación menstrual, la sexualidad, la espiritualidad o el énfasis en producciones artísticas; también aquí se está dando una diversificación. Respecto al liderazgo de los talleres, existe una gran diversidad, desde filosofías horizontales autogestionadas hasta una clara profesionalización (sin que estás opciones sean excluyentes entre sí): cada vez más personas se están especializando en ofrecer formaciones relacionadas con el ciclo. En este ámbito, tal como sucede en otras muchas iniciativas sociales y feministas, la tendencia a la profesionalización es evidente, extendiéndose a su vez dentro del feminismo lo que Esteban (2015) ha denominado la cultura experta. En los últimos años han destacado igualmente propuestas que tienen lugar a través de las redes sociales y la cultura virtual, ${ }^{8}$ algo que será fundamental analizar, siempre teniendo en cuenta la enorme diversidad presente en ella. ${ }^{9}$

\footnotetext{
${ }^{8}$ Entre otras propuestas, una experiencia interesante y de mucha influencia sería la de la comunidad virtual Soy1soy4, creada por la pedagoga menstrual Erika Irusta (véase https://soy1soy4.com/). Irusta ya desde antes daba talleres presenciales y ha producido diferentes contenidos en torno al tema (véase, entre otros, Irusta, 2018).

${ }^{9}$ Véase, entre otras, Ramírez, 2019.
} 


\subsection{Un ensamblaje de afectos, estrategias político-sensoriales y procesos de socialización para el cambio}

A través de estas propuestas críticas se configura lo que Carmen Díez Mintegui (1993: 175) denominó en su día socializaciones para el cambio, para dar a entender que, además de una socialización para la continuidad de la cultura hegemónica, se producen (o pueden producir) segundas socializaciones encaminadas a promover un cambio respecto a dicha cultura hegemónica. Esto es así cuando, por ejemplo, se discute la industria menstrual dominante y se experimenta una gestión alternativa de la sangre y de las vivencias del ciclo, a través de distintos procesos de reflexión. A medida que se problematiza la perspectiva negativa menstrual, emergen prácticas, afectos y vivencias encarnadas que facilitan esas nuevas socializaciones, dando lugar a otras maneras de habitar y ser cuerpos y mundo. Además, estas subjetividades individuales y colectivas en continua apertura y transformación, entendidas como un «conglomerado de territorios vivenciales y experienciales» donde hibridan «experiencias, deseos, relaciones, agencias y resistencias» (Diz, 2019: 94) permiten emerger otras maneras de hacer política feminista (Ahmed, 2004; Esteban, 2015).

El hecho de juntarse, sea en un taller organizado o no, conlleva procesos grupales muy interesantes. Luna cuenta cómo en su pueblo formaron un grupo de trabajo en torno al ciclo: «Pusimos carteles en la calle, [...] y empezamos a juntarnos y cada quince días alguien del grupo dinamizaba la sesión». Se juntaron durante un año en un local cedido por una asociación cultural y tomaron como punto de partida diferentes trabajos:

Fue una experiencia preciosa. Algunas nos conocíamos de vista, otras no, el primer día nos juntamos bastantes y luego ya la continuidad fue variando. En la primera sesión había una reunión en la sala contigua [...] nosotras partiéndonos de risa, y una amiga que estaba en la otra reunión, me dijo: «jo, dabais ganas de ir con vosotras». Sí, fue súper bonito. [...] cada una contaba cómo estaba viviendo el tema que habíamos elegido, y después hacíamos algunos ejercicios relacionados con ello, y lo compartíamos entre nosotras [...] hablábamos de nuestras experiencias y reflexionábamos (Luna, 2018).

Narra aquella experiencia con una gran sonrisa. Fue muy importante para las participantes, un proceso colectivo de conocimiento y concienciación. La percepción del propio cuerpo fue transformándose, pero también se crearon relaciones afectivas importantes entre ellas. 
Siguiendo a Esteban (2004), la dimensión corporal «es la base material y carnal de posibilidad para la acción individual y colectiva» y, por tanto, «también la base para la transformación social y cultural». Entender una acción social como un proceso afectivo y físico (Berardi, 2014; Esteban, 2015; Diz, 2019) ofrece, en mi opinión, el marco apropiado para investigar el lugar que tiene la colectividad, pero también las relaciones y los afectos en las políticas menstruales, entendiendo afecto en su sentido amplio y no solo en lo relativo a los sentimientos ni a los aspectos más cognitivos ni conscientes del campo emocional (Gould, 2009). Para muchas implicadas, junto a sus vivencias, sus referencias teóricas, su socialización feminista y sus ganas de experimentar y de buscar alternativas vitales transformadoras, las relaciones afectivas han sido un catalizador sumamente importante para profundizar en esos argumentos y comprometerse y para que se produzca esa socialización para el cambio de la que hablaba. Si bien durante la primera década del siglo XXI era posible encontrar información crítica relativa al tema (en redes sociales, fanzines, folletos...), no era tan abundante ni tan accesible, ya que hoy día es un campo fácilmente al alcance gracias a las redes sociales, los libros divulgativos, etc. Aún así y, según muchas informantes, especialmente cuando la información no era tan accesible, a menudo se han acercado al tema a través de sus amistades, amantes o compañeras de los círculos feministas cercanos. Por ello, al fijarnos en dichas propuestas y re-socializaciones, es importante observar las políticas de la intimidad, la influencia de los afectos y las relaciones en la creación y la difusión de los saberes, en la autogestión de la salud y en otro tipo de propuestas vitales.

Muchas informantes cuentan que las relaciones afectivas han sido el catalizador para profundizar en esos argumentos y producir esa socialización para el cambio, por ejemplo, en cuanto a la gestión del sangrado. Kris (26), que tiene un gran conocimiento sobre la autogestión de la salud, ha llegado a conocer distintas iniciativas gracias a sus amistades: una vez, organizaron una charla en la casa ocupada donde vivían, ya que su compañera de casa había profundizado el tema; otra vez, participó en un taller organizado por unas amigas. En el caso de Eva (25), que es educadora, a medida que ha ido trabajando sobre el tema, su experiencia respecto a los dolores menstruales ha ido transformándose. En ese trayecto, influyeron varias amigas, sobre todo su pareja de la universidad: 
Pues ya cuando lo compartes con la gente, y con la gente que lo lleva de otra forma. Por ejemplo, cuando conocí a Amaia en Gasteiz, nos hablaba de la Mooncup y de cosas así. Alize también... cuando conocí a Alize, también me estuvo hablando de lo mismo [se ríe]. Y esas cosas siempre ayudan mucho, no sé, conocer otras formas de llevarlo. Y luego ya cuando vivía en Madrid, pues algunas congelaban su sangre de la regla y tal... que si «[...] esto para las plantas es muy bueno», [...] o con la idea de emplearlo para alguna acción feminista [...] Yo creo que, en mi caso, más que interés ha sido más descubrir que hay otras formas y que hay inquietudes o quienes reivindicaban todo eso, formas alternativas de gestionarlo. Sobre todo, con Amaia, durante un tiempo fue ella quien... ella me enseñó muchas cosas. Y de ahí empecé a sentir la necesidad. Desde el feminismo (Eva, 2012).

En el caso de Maialen, en su politización de las vivencias menstruales tuvo mucha influencia la relación que iba tejiendo con su compañera de piso; comenzaron a repensar el ciclo, aprendiendo y experimentando juntas. Justamente, uno de los principales motores de este recorrido ha sido el placer experimentado, junto a la curiosidad y el compromiso. El deseo de investigar y de disfrutar que comparte con su compañera y las diversas lecturas que hacen juntas influyen directamente en este proceso de politizar la menstruación. Comenzaron a hacer talleres y a participar en otras iniciativas: «Teníamos mucha emoción, mucha curiosidad y ganas de investigar, una relación muy íntima $[. ..] \mathrm{y}$, sobre todo, fue un proceso muy placentero y vital en nuestras trayectorias de vida».

De hecho, tal como vemos en palabras de Maialen, otra de las conclusiones que revela esta investigación, y que está relacionada también en parte con los procesos afectivos que tienen lugar en ellos, se refiere a que, a medida que se ha cuestionado el estigma menstrual, las experiencias de placer han emergido con mucha intensidad. Desde una posición de repensar el placer desde una perspectiva crítica con las desigualdades sociales (Vance, 1989; Muelas, 2015; Guilló Arakistain, 2016), se han observado las diversas prácticas de placer relacionadas con la politización del ciclo menstrual que las informantes han explicitado, tales como 1) la sensación de placer en los procesos de empoderamiento corporal y su experimentación, 2) los procesos de disfrute y complicidad relacionados con momentos colectivos de construcción de conocimientos en torno al ciclo, o incluso 3) experiencias placenteras que surgen a través de habitar la subversión (Guilló Arakistain, en prensa). Así, en las políticas analizadas, la curiosidad, la creatividad, los afectos y el juego han sido componentes fundamentales de los itinerarios de placer que interpelan la mi- 
rada negativa de la biomedicina, mostrándose la vivencia del placer como una vía de implicación de los procesos de transformación social y epistemológica.

Sin embargo, dicho enfoque del placer no desestima el potencial transformador que otras emociones también tienen, ya que «la voluntad de cambio social y el rechazo del orden existente le debe mucho a sentimientos como la ira y el resentimiento» (Cabanas e Illouz, 2019). Se ha observado que la rabia, la preocupación, el dolor, la vergüenza, la tristeza o el propio desasosiego también han activado procesos de cuestionamiento y resignificación, que pueden ser además estímulo de estados altamente reflexivos, o activar el rechazo al orden existente y la voluntad de cambio social. La ira menstrual puede ser vital para cuestionar el dolor y los discursos sexistas, así como para generar procesos de subversión.

Al tiempo que el tipo de iniciativas es cada vez más variado, lo mismo sucede con las estrategias políticas, estéticas y sensoriales que en ellas surgen. De hecho, se ha observado que incluso en una misma propuesta pueden operar estrategias que producen imaginarios y evocaciones diferentes entre sí, donde convergen sensaciones muy diferentes. De entre toda la variedad de experiencias, cabe destacar lo que se puede denominar una estrategia política-sensorial y de los afectos que se está desarrollando en diferentes iniciativas y que he definido como la performatividad del asco (Guilló Arakistain, 2020a). Aunque aquí no me pueda extender en su análisis, es muy sugerente cómo algunas propuestas analizadas (como instalaciones artísticas o performances) explicitan el asco menstrual y juegan con su estética, justamente para interrogar, comprender e interpelar las causas de este, cuestionando la cultura de la negación del ciclo, la regulación biopolítica de los cuerpos y el sistema de género.

Tanto el asco, por su transgresión, como el placer, por su potencial creativo, nos pueden invitar a contrarrestar la mirada biomédica tan negativa del ciclo y a narrar lo menstrual de otra manera, ya sea de una manera subversiva, de reapropiación, o como elementos que cuestionan las posibilidades y posiciones a las que son capaces de acceder, transformar o reivindicar las diferentes corporalidades.

\subsection{Estrategias e ideologías feministas y de género}

Tal como he profundizado en otros trabajos (Guilló-Arakistain, 2020), la ideología de la normatividad menstrual se sustenta en las epistemologías 
de dimorfismo sexual y tiende a definir el ciclo como un proceso fisiológico universal y eje explicativo fundamental de la diferenciación corporal, dentro de una visión polarizada de los procesos hormonales, endocrinológicos y genitales, y vinculada exclusivamente a la función reproductiva (aunque el ciclo menstrual afecte a otros órganos y funciones). Sin embargo, la materialidad, las vivencias y las identidades corporales desafían dicha ideología normativa. Hay muchas mujeres que por muy diferentes motivos no tienen el ciclo menstrual, de la misma manera que algunos hombres trans y personas no binarias menstrúan..$^{10}$ La menstruación no es, por tanto, una verdad universal de los cuerpos considerados femeninos. Así, algunos cuerpos sufren de manera más intensa la violencia del sistema de género, al aflorar el conflicto con una visión dicotómica y rígida del paradigma biomédico. Pero no se trata de que algunos cuerpos no cumplan dicha normatividad (iqué cuerpos la cumplen?), sino de hacernos conscientes y responsabilizarnos respecto a la base estructural que sostiene las violencias que produce dicha normatividad, ya que esa ideología dominante es normativa y excluyente, refuerza las jerarquías entre cuerpos y, a su vez, tiende a homogeneizar la experiencia menstrual de distintas maneras.

Pero los diferentes discursos en torno al género han ido reformulándose. Aunque en algunos casos analizados se ha definido la menstruación como eje definidor de la feminidad, con una cierta sobre-enfatización del carácter positivo del ciclo, y como una estrategia claramente identitaria, algo que puede ser, en cierta manera, una vía para el empoderamiento, he podido observar que esta última década se han difundido perspectivas más inclusivas sobre los cuerpos menstruantes. Se ha problematizado cada vez más el dimorfismo sexual, mencionando a menudo términos como «mujeres sin menstruación» o «personas trans menstruantes», o a través de términos como «personas o cuerpos menstruantes». No obstante, y a pesar de los cambios, dada la cultura occidental biologicista en la que vivimos, se observan también riesgos claros de biologización en este campo, que son importantes de analizar desde los estudios críticos y feministas de la ciencia. Me refiero, por ejemplo, a los discursos sobre la ciclicidad y las hormonas. Las ideologías en torno a lo cíclico y las distintas fases menstruales que, aunque están presentes en muchas referencias clásicas, se han reforzado y propaga-

\footnotetext{
${ }^{10}$ Véanse, entre otros: Chrisler et al., 2016; Guilló-Arakistain, 2020; Rydström, 2020; etc.
} 
do mucho en los últimos años, son clave para mostrar y entender la variabilidad hormonal dentro de los cuerpos y, así, cuestionar la hiperproductividad que el sistema económico capitalista impone a los cuerpos, un cuestionamiento en el que Irusta (2018) y Gómez Nicolau y Marco Arocas (2020) profundizan. Sin embargo, de vez en cuando nos encontramos con lecturas algo cerradas de las distintas fases, con cierta dificultad para romper con la reproducción de los modelos explicativos hormonales biomédicos. Pueden ser estrategias que permitan una mejor comprensión de las propias vivencias y del dinamismo de los cuerpos y también un modo de valorar conocimientos antes negados y colonizados. Pero, cuando esto se interpreta de manera muy limitada, estática y rígida, entendiendo que dichas fases suceden de una forma absoluta en todos los cuerpos menstruantes, se pueden reproducir visiones naturalizadoras.

Se están creando nuevos marcos interpretativos. Un reto importante sería tener en cuenta que el proceso menstrual ocurre junto a otros procesos corporales y que las hormonas se producen de forma muy diferente y variable en cada cuerpo. Además, desde una perspectiva multicausal y dinámica de la biología (Martin, 1987; Valls Llobet, 2009; Esteban, 2001; Blázquez, 2005; FaustoSterling, 2006; García Dauder y Pérez Sedeño, 2017), no podemos entender el ciclo sin la influencia de los diversos condicionantes sociales, lo que nos lleva a complejizar todavía más la perspectiva de la ciclicidad. Así, es fundamental considerar las diferentes tendencias androcéntricas de la biomedicina (Valls Llobet, 2009; García Dauder y Pérez Sedeño, 2017), tanto el sesgo androcéntrico diferencialista desgranado en este apartado como el sesgo androcéntrico universalista que toma las experiencias masculinas como referente universal de las experiencias de todos los cuerpos, sin prestar atención a la morbilidad diferencial ni a la diversidad corporal, y así desarrollar una mirada compleja.

\section{RETOS EPISTEMOLÓGICOS, POLÍTICOS Y SOCIALES PARA LA TRANSFORMACIÓN SOCIAL}

Como hemos visto, asistimos a una reconfiguración plural de los cuerpos menstruantes. En este artículo se ha hecho una aproximación a las políticas y las culturas alternativas menstruales, a un cuerpo político feminista menstrual múltiple y subversivo, con sus diversos planteamientos feministas y de género, 
estrategias político-sensoriales y afectivas a través de la reflexión sobre los cambios significativos producidos. Se ha propuesto la importancia de analizar la importancia de las emociones, los afectos y los encuentros colectivos que activan (o pueden activar) nuevas socializaciones y estimulan desplazamientos en el pensamiento, proponiendo, también, nuevas maneras de estimular la reflexión y hacer política. Se emplean diversas estrategias, se politizan los espacios de seguridad y la construcción colectiva de saberes. Será interesante no perder de vista cómo se desarrollará en el futuro este incremento de propuestas, pero, sobre todo, será necesario atender a las consecuencias políticas, materiales, simbólicas y sociales, al igual que analizar quiénes serán sus sujetos políticos y las diversas formas que adoptarán en diferentes contextos. En muchas de las sociedades actuales la cultura neoliberal incide intensamente en las vivencias en torno a la salud, el cuerpo y la vida, fortaleciendo una idea muy individualista y productivista del cuerpo y del bienestar, obviando que no todas las personas tienen las mismas posibilidades para esta atención y dejando de lado las visiones críticas que cuestionan estos paradigmas de cuidado mediados por el consumo. Por ello, tener las desigualdades sociales y materiales presentes en la politización de la salud es un reto permanente.

Estas políticas y culturas están permeando algunas esferas sociales y contribuyen a explicitar las desigualdades sociales y de género. A pesar de las transformaciones analizadas, no se debe perder una mirada global: la visión general del ciclo menstrual sigue siendo negativa, las ganancias de las grandes corporaciones de compresas y tampones siguen creciendo espectacularmente (instrumentalizando el discurso feminista) y los diversos recursos (compresas, tampones, medicinas, etc.) no son accesibles para todas las personas que los necesitan (por ejemplo, para menstruantes en prisión, en situación de calle o en condiciones de pobreza). Poco o nada se investigan los dolores asociados al ciclo o los múltiples efectos que puede implicar más allá de la reproducción y, además, la industria farmacéutica tiene un peso enorme en dichas investigaciones. $\mathrm{Y}$, a pesar de los cambios, las rígidas normas de género siguen imperando y violentando a las personas, articuladas estrechamente con otras fuentes de opresión. ¿Qué oportunidades nos podría brindar la visibilidad emergente del tema en la actualidad para cuestionar y combatir las desigualdades sociales que siguen manteniéndose? Seguir revisando y trabajando a través de enfoques dinámicos del género, continuar repensando cómo colectivizar los procesos de politización de la 
salud desde una perspectiva interseccional y atendiendo en todo momento a las condiciones materiales de las personas y mantener una perspectiva crítica hacía la sociedad del consumo podrá ayudarnos a seguir fortaleciendo este cuerpo político y múltiple de la menstruación.

Los cuerpos resisten, subvierten y desestabilizan el paradigma biomédico hegemónico, transgrediendo las definiciones hegemónicas del cuerpo generizado. Es preciso poner en diálogo las prácticas corporales, los procesos afectivos y las reflexiones y saberes de las activistas y personas implicadas en el activismo menstrual con los conocimientos biomédicos, la teoría feminista y otras teorías sociales. Es a través de este diálogo, siempre inacabado y constante, como se podrán desarrollar pensamientos híbridos, críticos y alternativos, que contribuyan a afrontar, y esperemos que a superar, las desigualdades sociales y de género.

\section{BIBLIOGRAFÍA}

Ahmed, Sara (2004). The Cultural Politics of Emotion. London: Routledge.

Aterido Martín-Luengo, Irene (2018). La revolución roja: menstruación consciente desde los feminismos. Revista Mujeres y Salud Mys, 45, 1518.

Berardi, Franco "Bifo" (2014). Una sublevación colectiva es antes que nada un fenómeno físico, afectivo, erótico. Eldiario.es. Recuperado de: http://www.eldiario.es/interferencias/bifo-sublevacion-afectos_6 -31957806o.html [Consultado el 2 de enero de 2020].

Blázquez, María Isabel (2005). Aproximación a la antropología de la reproducción. AIBR. Revista de Antropología Iberoamericana, 42, 125 .

Blázquez, Maribel y Bolaños, Eva (2017). Aportes a una antropología feminista de la salud: el estudio del ciclo menstrual. Salud Colectiva 13(2), 253-265. doi: 10.18294/sc.2017.1204

Bobel, Chris (2010). New Blood: Third-Wave Feminism and the Politics of Menstruation. New Brunswick/New Jersey/London: Rutgers University Press. 
Bobel, Chris (2015). The year the period went public. Gender \& Society. Recuperado de: https://gendersociety.wordpress.com/2015/11/12/theyear-the-period-went-public/_[Consultado el 2 de enero de 2020].

Bobel, Chris, Winkler, Inga T., Fahs, Breanne, Hasson, Katie Ann, Kissling, Elizabeth Arveda y Roberts, Tomi-Ann (eds.) (2020). The Palgrave Handbook of Critical Menstruation Studies. Singapore: Palgrave Macmillan.

Cabanas, Edgar e Illouz, Eva (2019). Happycracia. Cómo la ciencia y la industria de la felicidad controlan nuestras vidas. Barcelona: Planeta.

Chrisler, Joan C., Gorman, Jennifer A., Manion, Jen, Murgo, Michael, Barney, Angela, Adams-Clark, Alexis, Newton, Jessica R. y McGrath, Meaghan (2016). Queer Periods: Attitudes Toward and Experiences with Menstruation in the Masculine of Center and Transgender Community. Culture Health, \& Sexuality, 18(11), 1238-1250. doi: https://doi.org/10.1080/13691058.2016.1182645

Díez Mintegui, Carmen (1993). Estudio comparativo de las relaciones de género en la comarca Donostialdea y la Ribera de Navarra, partiendo de la forma diferente de participación de mujeres y hombres en las actividades laborales. Tesis doctoral. Donostia: UPV/EHU.

Diz, Carlos (2019). Maneras de vivir: emoción, política e identidades en movimiento. ANDULI, Revista Andaluza de Ciencias Sociales, 18, 93-117. doi: https://doi.org/10.12795/anduli.2019.i18.05

Ehrenreich, Barbara (2011). Sonríe o muere. La trampa del pensamiento positivo. Madrid: Turner.

Epelde, Edurne, Aranguren, Miren y Retolaza, Iratxe (2015). Gure Genealogia Feministak. Euskal Herriko Mugimendu Feministaren Kronika Bat. Andoain: Asociación Emagin.

Esteban, Mari Luz (2001). Re-producción del cuerpo femenino. Discursos y prácticas acerca de la salud. Donostia: Gakoa-Hirugarren Prentsa.

Esteban, Mari Luz (2004). Antropología del cuerpo. Género, itinerarios corporales, identidad y cambio. Barcelona: Bellaterra.

Esteban, Mari Luz (2011). Cuerpos, políticas feministas y agencia: el feminismo como cuerpo. En Villalba, Cristina y Álvarez, Nacho (eds.). 
Cuerpos politicos y agencia. Reflexiones feministas sobre cuerpo, trabajo $y$ colonialidad (45-84). Granada: Universidad de Granada.

Esteban, Mari Luz (2015). La reformulación de la política, el activismo y la etnografía. Esbozo de una antropología somática y vulnerable. Ankulegi, Revista de Antropología Social, 19, 75-93.

Fahs, Breanne (2016). Out for blood, essays on menstruation and resistance. Albany: University of New York Press.

Fausto-Sterling, Anne (2006). Cuerpos sexuados: la política de género y la construcción de la sexualidad. Barcelona: Melusina.

Felitti, Karina (2016). El ciclo menstrual en el siglo XXI. Entre el mercado, la ecología y el poder femenino. Sexualidad, salud y sociedad. Revista Latinoamericana, 22, 175-206. doi: https://doi.org/10.1590/1984-6487.sess.2016.22.08.a

García Dauder, S. y Pérez Sedeño, Eulalia (2017). Las 'mentiras' científicas sobre las mujeres. Madrid: Catarata.

Gómez Nicolau, Emma y Marco Arocas, Elisabet (2020). Desafiando las reglas: articulaciones políticas del activismo menstrual. Revista Española de Sociología, 29 (3, supl. 1), 155-17o. doi: https://doi.org/10.2232 $5 / \mathrm{fes} / \mathrm{res} .2020 .62$

Gould, Deborah B. (2009). Moving politics: emotion and act up's fight against AIDS. Chicago: The University of Chicago Press.

Guilló Arakistain, Miren (2013). La incorporación de la investigación: políticas de la menstruación y cuerpos (re)productivos. Revista Nómadas, 39, 233-245.

Guilló Arakistain, Miren (2014). Mujeres jóvenes y menstruación: contracultura y resignificación del ciclo menstrual en el País Vasco. En Romaní, Oriol y Casadó, Lina (eds.). Jóvenes, desigualdades y salud. Vulnerabilidad y políticas publicas (143-165). Tarragona: Publicacions Univesitat Rovira i Virgili.

Guilló Arakistain, Miren (2016). Festaren analisi eta politika feministak. Ainguraketak eta erronkak. En Guilló Arakistain, Miren (ed.). Festak, genero-harremanak eta feminismoa. Begirada teoriko eta 
antropologikoak, praktika sortzaileak eta plazeraren kudeaketa kolektiboak (7-32). Bilbao: Udako Euskal Unibertsitatea (UEU).

Guilló Arakistain, Miren (2018). Habitar lo imponderable. Espiritualidades contemporáneas y lecturas alternativas de la menstruación. En Esteban, Mari Luz y Hernández, Jone M. (eds.). Etnografías feministas. Una mirada al siglo XXI desde la antropología vasca (183-207). Barcelona: Bellaterra.

Guilló Arakistain, Miren (2020a). Hilekoaren politika eta kultura alternatiboen etnografia bat: genero-konfigurazioak, gorputz-ahalduntzea eta ezagutza kolektiboak. Tesis doctoral. Donostia: UPV/EHU.

Guilló Arakistain, Miren (2020b). Una aproximación etnográfica a las relaciones entre espiritualidad, género y salud. Quaderns de l'Institut Català d'Antropologia, 36 (1), 21-36.

Guilló Arakistain, Miren (en prensa). Placer, agencia y menstruación: subversión y conocimientos colectivos para la transformación social. AIBR. Revista de Antropología Iberoamericana.

Guilló-Arakistain, Miren (2020). Challenging menstrual normativity: nonessentialist body politics and feminist epistemologies of health. En Bobel, Chris et al. (eds.). The Palgrave Handbook Of Critical Menstruation Studies (869-883). Singapore: Palgrave Macmillan. doi: https://doi.org/10.1007/978-981-15-0614-7_63

Hester, Helen (2018). Xenofeminismo. Tecnologías de género y políticas de reproducción. Buenos Aires: Caja Negra Editora.

Illouz, Eva (2014). El futuro del alma y la creación de estándares emocionales. Madrid: Katz Editores.

Irusta, Erika (2018). Yo menstrúo: un manifiesto. Barcelona: Catedral.

Kissling, Elizabeth A. (2006). Capitalizing on the Curse. The Business of Menstruation. Colorado/London: Rienner.

Layne, Linda L. (2010). Introduction. En Layne, Linda, Vostral, Sharra y Boyer, Kate (eds.). Feminist Technology (1-35). Urbana/Chicago/ Springfield: University of Illinois Press.

Martin, Emily (1987). The Women in the Body: A Cultural Analysis of Reproduction. Boston: Beacon Press. 
Muelas, Laura (2015). El placer como proceso creativo en la transformación feminista. Trabajo Fin de Máster. Donostia: Universidad del País Vasco.

Nogueiras, Belén (2013). Discursos y prácticas feministas en el ámbito de la salud en España (1975-2013). Trabajo Fin de Máster. Madrid: Universidad Complutense de Madrid.

Persdotter, Josephin (2013). Countering the menstrual mainstream. A study of the European Menstrual Countermovement. Master thesis. Gothenburg: University of Gothenburg.

Przybylo, Ela y Fahs, Breanne (2020). Empowered Bleeders and Cranky Menstruators: Menstrual Positivity and the "Liberated" Era of New Menstrual Product Advertisements. En Bobel, Chris et al. (eds.). The Palgrave Handbook of Critical Menstruation Studies (375-394). Singapore: Palgrave Macmillan. doi: https://doi.org/10.1007/978-981-15-0614-7-30

Ramírez, María del Rosario (2016). Del tabú a la sacralidad: la menstruación en la era del sagrado femenino. Revista Ciencias Sociales y Religión/Ciências Sociais e Religião. 18(24), 134-152. doi: https://doi.org/10.22456/1982-2650.62531

Ramírez, María del Rosario (2019). Menstrual cyber-activism: feminism in social networks. PAAKAT: Revista de Tecnología y Sociedad, 17 (9). doi: http://dx.doi.org/10.3287o/Pk.a9n17.438.

Rydström, Klara (2020). Degendering Menstruation: Making Trans Menstruators Matter. En Bobel, Chris et al. (eds.). The Palgrave Handbook of Critical Menstruation Studies (945-959). Singapore: Palgrave Macmillan. doi: https://doi.org/10.1007/978-981-15-06147_68

Taboada, Leonor (1978). Cuaderno Feminista. Introducción al Self-Help. Barcelona: Fontanella.

Tarzibachi, Eugenia (2017). Cosa de Mujeres. Menstruación, género y poder. Buenos Aires: Sudamericana.

Thiébaut, Élise (2018). Esta es mi sangre. Pequeña historia de la(s) regla(s), de las que la tienen y de los que las marcan. Xixón: Hoja de Lata. 
Valls Llobet, Carmen (2009). Mujeres, salud y poder. Barcelona: Cátedra. Vance, Carole S. (1989). El placer y el peligro: hacia una política de la sexualidad. En Carole S. Vance (ed.). Placer y peligro. Explorando la sexualidad femenina (9-49). Madrid: Talasa. 